\title{
Correction: mechanisms of Legionella pneumophila-induced interleukin-8 expression in human lung epithelial cells
}

Hiromitsu Teruya ${ }^{1,2}$, Futoshi Higa ${ }^{2}$, Morikazu Akamine ${ }^{2}$, Chie Ishikawa ${ }^{1,3}$, Taeko Okudaira ${ }^{1,4}$, Koh Tomimori ${ }^{1,2}$, Naofumi Mukaida ${ }^{5}$, Masao Tateyama ${ }^{2}$, Klaus Heuner ${ }^{6}$, Jiro Fujita ${ }^{2}$ and Naoki Mori ${ }^{1 *}$

\section{Correction}

After the publication of this work [1], we became aware of the fact that $\beta$-actin control images in Figures two $(\operatorname{dot} O$ mutant), three $\mathrm{A}$, eight $\mathrm{A}$ and nine $\mathrm{A}$ (figures 1,2 , 3 and 4 in this manuscript, respectively) were duplicated. The last author, Naoki Mori takes full responsibility for these errors in the original article. We repeated the experiments, and all the Figures mentioned above were deleted and new data substituted. The conclusions from the figures are not altered in any way. We regret any inconvenience that this inaccuracy in the original data might have caused.

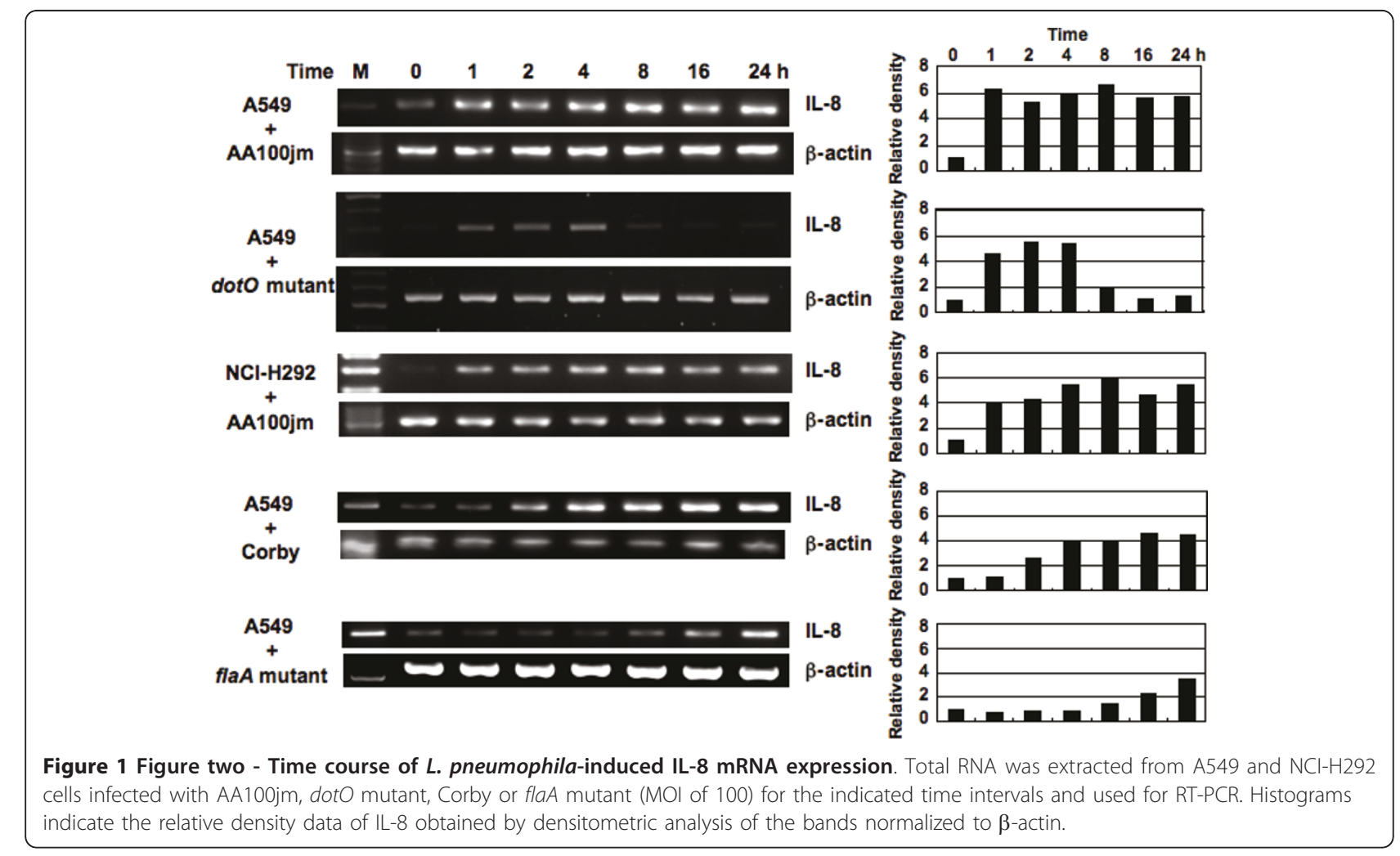

* Correspondence: n-mori@med.u-ryukyu.ac.jp

'Division of Molecular Virology and Oncology, Graduate School of Medicine,

University of the Ryukyus, 207 Uehara, Nishihara, Okinawa 903-0215, Japan 

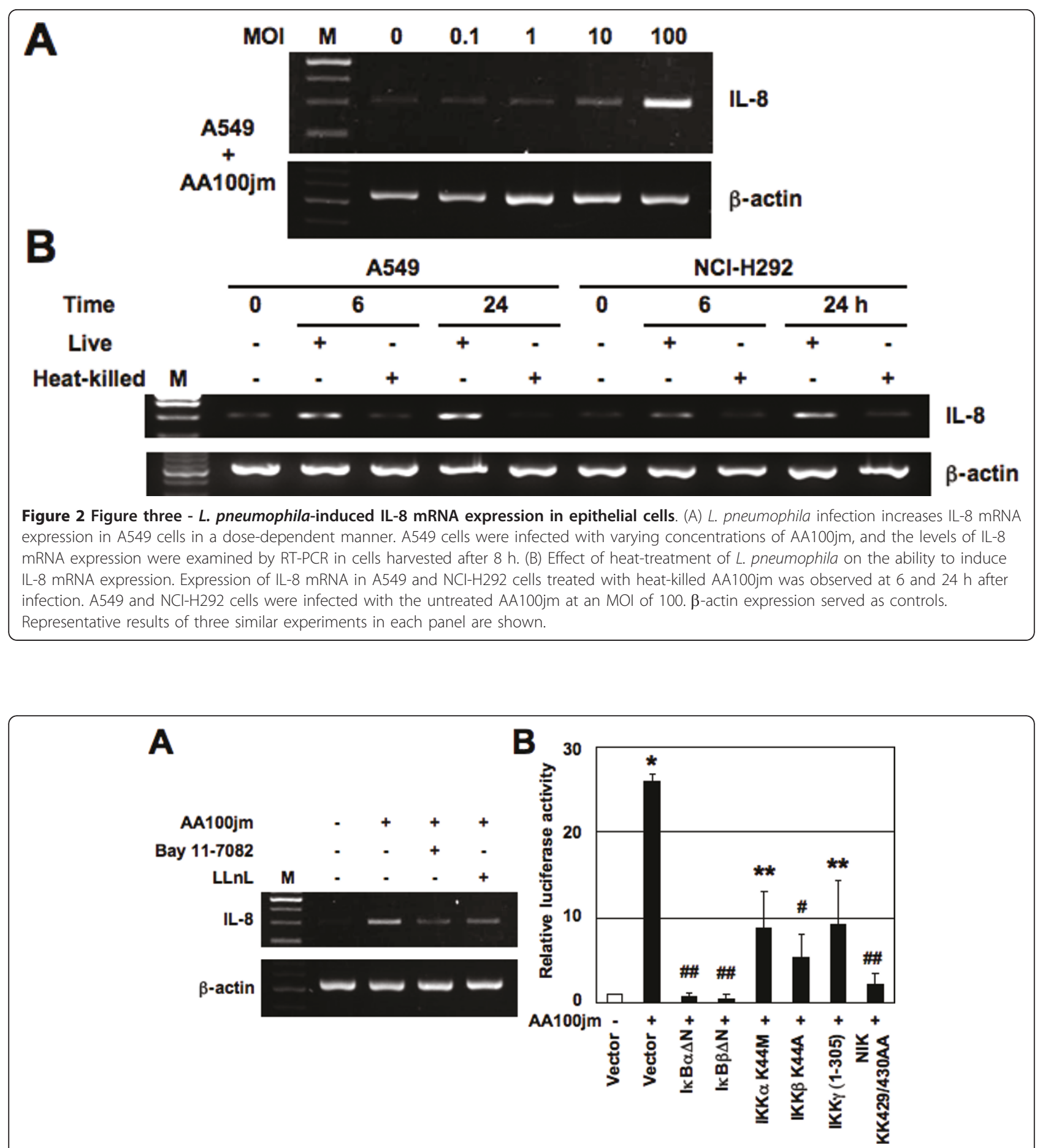

Figure 3 Figure eight - NF- $\kappa$ B signal is essential for activation of IL-8 expression by L. pneumophila. (A) Bay 11-7082 and LLnL inhibit IL-8

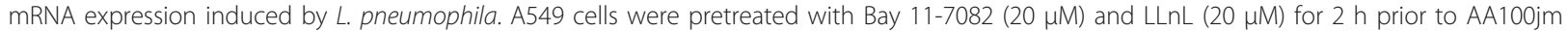
infection. They were subsequently infected with L. pneumophila for 6 h. IL-8 mRNA expression on harvested cells was analyzed by RT-PCR. Representative results of three similar experiments in each panel are shown. (B) Functional effects of $|\kappa B \alpha,| \kappa B \beta$ and IKK dominant interfering mutants and kinase-deficient IKK $\alpha$, IKK $\beta$ and NIK mutants on L. pneumophila-induced activation of the IL-8 promoter. A549 cells were transfected with $40 \mathrm{ng}$ of -1481-luciferase construct and $2 \mu \mathrm{g}$ of the indicated mutant plasmids or empty vector (pCMV4), and then infected with $L$. pneumophila (MOI of 100) for $48 \mathrm{~h}$. Open bar represents luciferase activity of empty vector without L. pneumophila infection. All values were first calculated as a fold induction relative to the basal level measured in uninfected cells. Data are mean \pm SD values of three independent experiments. ${ }^{*}, P<0.0005$ (compared to uninfected cells). ${ }^{*}, P<0.05 ; \#, P<0.01 ; \# \#, P<0.005$ (compared to cells transfected with empty vector with further L. pneumophila infection). 


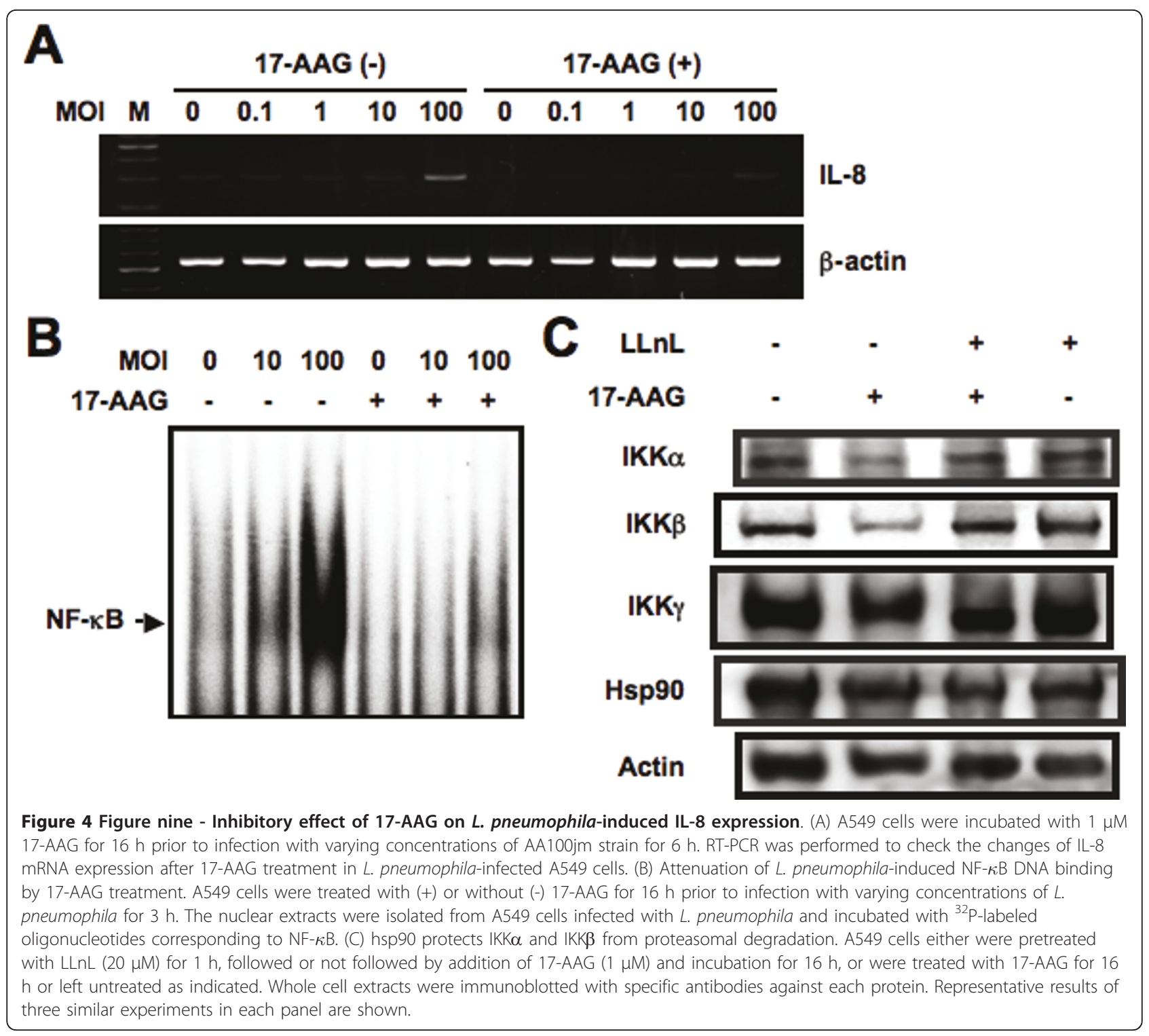

\section{Author details}

'Division of Molecular Virology and Oncology, Graduate School of Medicine, University of the Ryukyus, 207 Uehara, Nishihara, Okinawa 903-0215, Japan. ${ }^{2}$ Division of Control and Prevention of Infectious Diseases, Graduate School of Medicine, University of the Ryukyus, 207 Uehara, Nishihara, Okinawa 9030215, Japan. ${ }^{3}$ Division of Child Health and Welfare, Faculty of Medicine, University of the Ryukyus, 207 Uehara, Nishihara, Okinawa 903-0215, Japan. ${ }^{4}$ Division of Endocrinology and Metabolism, Faculty of Medicine, University of the Ryukyus, 207 Uehara, Nishihara, Okinawa 903-0215, Japan. ${ }^{5}$ Division of Molecular Bioregulation, Cancer Research Institute, Kanazawa University, 13-1 Takara-machi, Kanazawa 920-0934, Japan. ${ }^{6}$ Institute for Molecular Infection Biology, Universitat Wuerzburg, Roentgenring 11, 97070 Wuerzburg, Germany.
Reference

1. Teruya H, Higa F, Akamine M, Ishikawa C, Okudaira T, Tomimori K, Mukaida N, Tateyama M, Heuner K, Fujita J, Mori N: Mechanisms of Legionella pnumophila-induced interleukin-8 expression in human lung epithelial cells. BMC Microbiol 2007, 7:102.

doi:10.1186/1471-2180-11-136

Cite this article as: Teruya et al: Correction: mechanisms of Legionella pneumophila-induced interleukin-8 expression in human lung epithelial cells. BMC Microbiology 2011 11:136 